Revue internationale d'éducation de Sèvres

34 | décembre 2003

La formation professionnelle initiale: une question de société

\title{
Portugal : réorganisation de l'enseignement et décentralisation
}

\section{Cécile de Bouttemont}

\section{OpenEdition}

\section{Journals}

Édition électronique

URL : https://journals.openedition.org/ries/1560

DOI : $10.4000 /$ ries. 1560

ISSN : 2261-4265

Éditeur

France Education international

Édition imprimée

Date de publication : 1 décembre 2003

Pagination : $15-20$

ISBN : 978-2-8542-0558-9

ISSN : $1254-4590$

Référence électronique

Cécile de Bouttemont, «Portugal : réorganisation de l'enseignement et décentralisation », Revue internationale d'éducation de Sèvres [En ligne], 34 I décembre 2003, mis en ligne le 11 avril 2012, consulté le 05 juillet 2021. URL : http://journals.openedition.org/ries/1560 ; DOI : https://doi.org/ $10.4000 /$ ries. 1560

Ce document a été généré automatiquement le 5 juillet 2021

(c) Tous droits réservés 


\title{
Portugal : réorganisation de l'enseignement et décentralisation
}

\author{
Cécile de Bouttemont
}

1 En dépit de progrès remarquables à plusieurs titres ${ }^{1}$, le système éducatif portugais reste confronté à des difficultés persistantes. Ainsi, selon une étude gouvernementale publiée en mars 2003, le taux d'abandon scolaire reste le plus élevé d'Europe : en 2001, un quart des jeunes de dix-huit à vingt-quatre ans n'étaient pas allés au terme des neuf années de scolarité obligatoire ${ }^{2}$. Par ailleurs, on constate des dysfonctionnements entre les nouveaux besoins du marché du travail et l'offre traditionnelle de diplômés ${ }^{3}$.

2 En prenant acte, le gouvernement portugais a fait de la lutte contre l'abandon scolaire ${ }^{4}$ et de l'insertion des jeunes sur le marché du travail européen ses priorités. Ces objectifs s'inscrivent dans le cadre de réformes mises en place progressivement autour de deux grands axes : la refonte et la réorganisation des enseignements ainsi que la réforme du mode de gestion des écoles lié au processus de décentralisation initié par la Constitution.

\section{Refonte et réorganisation des enseignements}

3 Fin mai 2003, le gouvernement a proposé une réforme de la loi de base de l'Éducation qui régit le système éducatif depuis 1986. Cette réforme, dont l'objectif est de lutter contre l'abandon scolaire, propose un allongement de la durée de la scolarité obligatoire de neuf à douze ans et une réorganisation de l'enseignement de base et de l'enseignement secondaire qui dureraient six ans ${ }^{5}$. Actuellement examinée au Parlement, elle est supposée entrer en application en 2005.

Parallèlement, des innovations ont été envisagées en ce qui concerne les contenus. Dans l'enseignement de base ${ }^{6}$, l'initiation aux langues étrangères à l'école primaire et l'introduction d'une deuxième langue obligatoire en septième année ${ }^{7}$ sont l'un des volets importants de cette réforme. La réforme des curricula de l'enseignement secondaire, dont l'entrée en vigueur est prévue pour la rentrée 2004-2005, tient à faire une place importante à l'enseignement des nouvelles technologies et à l'enseignement 
des langues. Les NTIC (nouvelles technologies de l'information et de la communication) deviendraient une discipline obligatoire à partir de la neuvième année. En revanche, la littérature portugaise, traitée indépendamment de la langue portugaise, deviendrait optionnelle à partir des classes de seconde et première. Cette mesure suscite une forte opposition de la part des professeurs de lettres ${ }^{8}$.

Le gouvernement envisage en outre d'introduire un examen de mathématiques et de langue portugaise dès la neuvième année. La réintroduction d'examens nationaux et la création d'une commission pour le développement des mathématiques, des sciences et de la langue portugaise montre la volonté du gouvernement d'améliorer la qualité de l'éducation.

6 Enfin, la restructuration des contenus de l'enseignement professionnel est également abordée dans le but de lutter contre l'abandon scolaire et de favoriser l'intégration des jeunes sur le marché du travail européen. Basé sur le développement de compétences qualifiantes et non plus sur l'acquisition de connaissances, il pourra bénéficier, en outre, de la création de centres de soutien scolaire durant toute la scolarité obligatoire.

\section{Décentralisation et évolution du mode de gestion des écoles}

7 La refonte et la réorganisation des enseignements s'appuient sur la prise en compte progressive du contexte socio-économique dans lequel s'insèrent les écoles. Ainsi le gouvernement promeut l'idée que l'école doit construire son autonomie à partir de la communauté qui l'entoure. Inscrite dans la Constitution après cinquante années de centralisation éducative, la décentralisation a été freinée faute de textes réglementaires, en raison des hésitations des organes centraux à céder leurs pouvoirs de décision tout comme des réticences des communautés locales à recevoir de nouvelles responsabilités sans se voir garantir les ressources nécessaires à leur exercice ${ }^{9}$.

Cependant, les trois dernières années ont vu se développer de nouvelles actions dans le prolongement de cette tendance. Ainsi, au printemps 2003, ont été créés les «conseils locaux d'éducation " ${ }^{10}$, inscrits dans la loi depuis 1999 en tant que nouvelles instances d'administration et d'appui relevant des municipalités. Ces conseils doivent permettre aux municipalités ainsi qu'à tous les partenaires sociaux d'intervenir dans la définition de l'offre en éducation sur leur territoire en termes stratégiques par rapport à leur propre politique de développement local. Les municipalités sont également chargées de l'élaboration de la charte scolaire. Toutefois, la légitimité des décisions prises par ces organes, qui restent consultatifs, est fortement remise en cause par les enseignants.

Par ailleurs, et toujours dans le cadre de la décentralisation éducative, le nouveau régime juridique de l'autonomie de l'administration et de la gestion des établissements, entériné par le décret-loi de 1998, est entré en vigueur en 2000. Il crée pour chaque établissement un organe de participation de la communauté locale, l'assemblée d'école, chargée de définir les grandes orientations de l'établissement. Jusqu'à cette nouvelle réglementation, les professeurs, cooptant le chef d'établissement, étaient majoritaires dans cette structure. Ils le restent encore mais les parents et les personnels non enseignants peuvent désormais participer à l'élection de l'équipe de direction. Cette nouvelle mesure ne semble pas faire l'unanimité chez les enseignants. 
10 Malgré les difficultés, les mesures récentes montrent la volonté politique du gouvernement d'avancer dans la voie de la décentralisation.

\section{NOTES}

1. «La scolarisation des enfants et des adolescents a connu un développement exponentiel. Aujourd'hui dans la pratique, chaque génération est intégrée à $100 \%$ dans l'enseignement de base [...] indicateur tout à fait comparable aux moyennes statistiques européennes. [...] Le parc scolaire a augmenté de façon spectaculaire [...] » in Joao Barroso et al., Analyse de l'évolution des modes de régulation institutionnalisée dans le système éducatif du Portugal, mars 2002, p. 9, Faculté de Psychologie et de Sciences de l'Education, Lisbonne.

2. Dépêche AFP, 28 mai 2003.

3. Joao Barroso et al., op. cit, p. 10.

4. Sophie Condat, Bernadette Plumelle, «La lutte contre l'abandon scolaire», Revue internationale d'éducation de Sèvres, mars 2003, n 32, p. 12.

5. Le Monde - La Lettre de l'Éducation, $\mathrm{n}^{\circ}$ 418, 9 juin 2003.

6. Isabel Barreno et Catherine Clément, "L'enseignement des langues vivantes au Portugal», Revue internationale d'éducation de Sèvres, juin 2003, p. 49.

7. L'enseignement de base compte actuellement neuf années.

8. AEF, janvier 2003.

9. Joao Barroso et al., op. cit. p. 18.

10. AEF, avril 2003.

INDEX

Mots-clés : système éducatif

Index géographique : Portugal

\section{AUTEUR}

\section{CÉCILE DE BOUTTEMONT}

Documentaliste, centre de ressources documentaires du CIEP 